\title{
Satisfactory long-term results after simultaneous resection of the esophagus, stomach and pancreas
}

\author{
Orlin Belyaev • Christophe A. Muller • Waldemar Uhl
}

Received: 28 March 2008 / Accepted: 11 July 2008 / Published online: 15 August 2008

(C) The Author(s) 2008

\begin{abstract}
Purpose Surgical procedures for esophageal cancer and chronic pancreatitis are demanding and still characterized by high morbidity and mortality. Cases of one-stage combined operations on the pancreas and esophagus with a long-term follow-up are rarely reported.

Materials and methods We present the case of a 54-year-old woman with a Barrett's carcinoma and an 8-year history of severe chronic pancreatitis complicated with multiple episodes of cholangitis and resistant to conservative treatment. Results After a successful one-stage operation consisting of esophagogastrectomy and pancreaticoduodenectomy, a 30-month disease-free follow-up with a good quality of life has been observed.

Conclusion Complicated surgical procedures such as onestage multiple organ resections may offer, in selected cases, satisfactory long-term results, provided that patients are treated at a high-volume center by a multidisciplinary team.
\end{abstract}

Keywords Chronic pancreatitis · Barrett's carcinoma .

Pancreatoduodenectomy $\cdot$ Esophageal resection $\cdot$ Follow-up

\section{Introduction}

Synchronous surgical diseases of the esophagus and pancreas are rarely reported. There are few reports of double primary cancers of both organs, either synchronously existing, or metachronously appearing [1-5]. To our

O. Belyaev · C. A. Muller • W. Uhl $(\bowtie)$

Department of General Surgery, St. Josef Hospital,

Ruhr University Bochum,

Gudrunstrasse 56,

44791 Bochum, Germany

e-mail: w.uhl@klinikum-bochum.de knowledge, there is no case report in the literature, describing the successful treatment by means of a onestage surgical procedure of co-existing severe chronic pancreatitis and esophageal cancer, combined with a longterm follow-up of the patient.

\section{Case report}

A 54-year-old woman with severe recurrent chronic pancreatitis and multiple episodes of cholangitis over the last 8 years was scheduled for elective surgery on the pancreatic head. Surgery was indicated since multiple stenting of the common bile duct (CBD) and the pancreatic duct failed to alleviate her symptoms and cholangitis was difficult to further control conservatively despite aggressive antibiotic treatment. The patient, who was $170 \mathrm{~cm}$ tall, had reduced her body weight from $70 \mathrm{~kg}$ to $57.5 \mathrm{~kg}$; that meant a BMI reduction from $24.5 \mathrm{~kg} / \mathrm{m}^{2}$ to $20.1 \mathrm{~kg} / \mathrm{m}^{2}$ for a period of 12 months. She suffered no diabetes and had a normal oral glucose tolerance test and HbA1c values. The stool elastase level of $12 \mu \mathrm{g} / \mathrm{kg}$ was pathologically low. The patient took regularly pancreatic enzymes as a substitution therapy for her exocrine pancreatic insufficiency and a betablocker for her arterial hypertension. She reported being a heavy smoker for more than 30 years and had recently suffered a deep venous thrombosis of the lower extremities. At the time of her admittance, the patient had already four stents in the CBD and one stent in the pancreatic duct. Routine preoperative endoscopy found a Barrett's carcinoma at 36 to $39 \mathrm{~cm}$ from the dental line, which was staged by the endoscopic ultrasound as an uT3uN1 tumor.

A one-stage combined multiorgan resection was performed on November 02, 2005. A median laparotomy was performed. Exploration revealed severe chronic inflamma- 


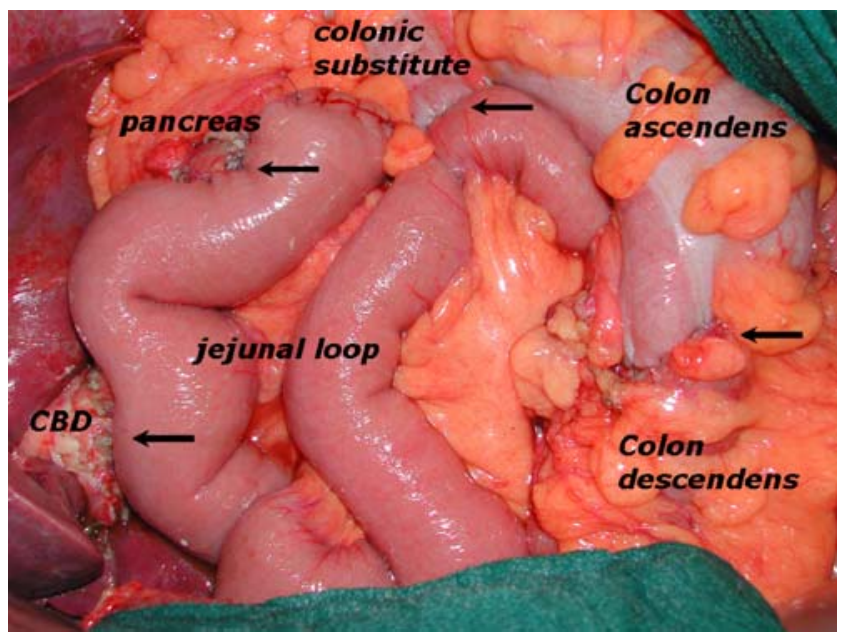

Fig. 1 Final intraabdominal view (from the side of the operating surgeon) after completed reconstruction. $C B D$ common bile duct. Arrows indicate the four intraabdominal anastomoses

tory changes in the upper abdomen with massive adhesions and fibrotic changes and a large inflammatory mass in the head of the pancreas. The posterior wall of the stomach was extremely adherent to the pancreatic head and isthmus. The esophageal tumor was palpated as a $2-3-\mathrm{cm}$ large lesion in the cardia. Because of the preoperative staging $\mathrm{uT} 3 \mathrm{uN} 1$ and the severe chronic changes in the anatomy of the stomach with compromised blood vessels, a formation of a gastric tube as a substitute was assessed not feasible and it was decided to perform a gastrectomy. Cholecystectomy and appendectomy were also performed. The right gastric, supraduodenal, and right gastroepiploic artery were divided, in that order, and the colon was totally mobilized. The duodenum was divided $4 \mathrm{~cm}$ below the pyloric ring. Lymph nodes in the liver hilus and around the celiac trunk were totally removed. The left gastric artery was then divided. The distal esophagus was carefully dissected free. The cranial endosonographic border of the tumor had been endoscopically marked with clips, palpable $5 \mathrm{~cm}$ under the tracheal bifurcation. A totally transabdominal operation on the Barrett's esophagus was assessed not feasible. Interposition of small bowel was impossible due to the fatty short mesentery of the jejunal loops. A right thoracotomy was performed and the distal part of the esophagus with the stomach was brought up into the thoracic cavity. The esophagus was divided above the level of the tracheal bifurcation. The middle and right colic vessels were then divided close to their roots. The ascending colon was divided just distally to the supply region of the ileocolic artery and an isoperistaltic colonic substitute supplied by the inferior mesenteric vein through a strong Riolan's arcade was brought up in the thoracic cavity and anastomosed to the esophagus remnant in the fashion of an endto-side esophagocolostomy. The pancreatoduodenectomy followed: the common bile duct was opened and a large volume of putrid bile, sludge and food remnants were evacuated under pressure from its lumen. Four plastic stents were taken out of the duct. The pancreatic duct was full with stones in distal direction, which were smashed using a pancreatic duct probe and flushed out of the duct. Reconstruction included an end-to-side duct-to-mucosa pancreaticojejunostomy and an end-to-side hepaticojejunostomy. The descending colon was divided just distally to the left colic artery and the formed distal end of the substitute was anastomosed to the jejunum in the fashion of an endto-side colojejunostomy $50 \mathrm{~cm}$ distal to the hepaticojejunostomy. The ascending and descending colon were then anastomosed end-to-end in front of the small intestinal loops. Figure 1 shows the final intraoperative view after completed reconstruction and Fig. 2 is a schematic presentation of the performed operation. The operative time was $9 \mathrm{~h}$, blood loss-300 ml.

The final histological staging of the Barrett's carcinoma differed from the endoscopic ultrasound staging and it was: pT1pN0(0/54)M0L0V0R0.

The postoperative course was uneventful. After $72 \mathrm{~h}$ at the ICU, the patient was transferred to the periphery. A

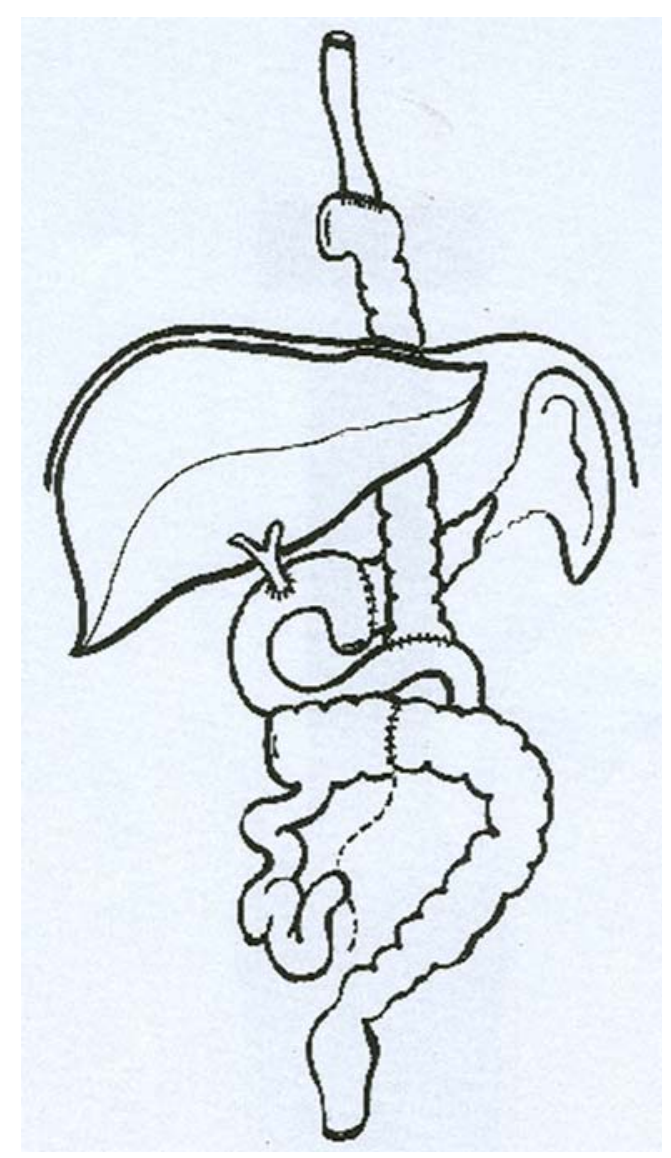

Fig. 2 Schematic presentation of the completed reconstruction in the thoracic and abdominal cavities 
stepwise unproblematic reduction of the analgesics (metamizol, sufentanyl and dipidolor) was achieved in the first postoperative days. A standard postoperative prophylaxis with octreotide $200 \mu \mathrm{g}$ three times a day for a week was applied in order to avoid pancreatic leakage. A double antibiotic combination of metronidazol and a cephalosporine third generation, and an antifungal drug was applied according to the results of the bile antibiogram. Oral fluid intake was started on the first and oral feeding-on the fourth postoperative day and was well tolerated. No diabetes mellitus developed postoperatively. The exocrine pancreatic insufficiency remained unchanged as expected with levels of stool elastase close to zero. Substitution with vitamins $\mathrm{A}, \mathrm{D}, \mathrm{E}, \mathrm{K}$ and $\mathrm{B}_{12}$ was performed at regular intervals. Neither systemic nor local complications developed during the hospital stay. The patient was discharged on the 28th day. At present, 30 months after surgery, the patient is doing well. The regular follow-up visits to our clinic show that the patient is in a good general condition, pain-free, with a reduced, but stable body mass index of about $18 \mathrm{~kg} / \mathrm{m}^{2}$, and without evidence of tumor recurrence. She visits our clinic regularly for routine control examinations.

\section{Discussion}

It is interesting to note that the Barrett's carcinoma was not detected earlier, despite the numerous consecutive endoscopic examinations and stenting procedures. A good longterm prognosis in our case is due to the benign character of the pancreatic disease and the early stage of the esophageal cancer-T1N0M0L0V0R0. It is noteworthy that the preoperative staging via endoscopic ultrasound assessed the tumor as much more advanced (uT3uN1) which was one of the reasons for performing a radical gastrectomy. The early histological stage of the tumor allowed a radical curative resection with the expectance of long-term survival. A resectional procedure for the chronic pancreatitis was obviously the last option to control the severe pain and recurrent cholangitis, after failure of all conservative measures. Although a duodenum-preserving pancreatic head resection was preoperatively discussed as an option, it became intraoperatively clear that the presence of five stents in the pancreatic and bile ducts, as well as the severe cholangitis could be successfully and reliably managed only by means of a Whipple procedure. In principle, the same surgical technique may be applied to synchronous early cancer of the esophagus and pancreatic head. However, the benefit of such extended surgery for two of the most sinister malignancies is hard to estimate since the number of such cases is too small. We want to pay attention to the fact that no operation-related morbidity appeared in that case, although colon replacement was used. According to the majority of authors, colon replacement should be justified only in cases where the stomach is not available [1, 4]. Furthermore, it is thought that the use of colon is more time consuming, invasive and entails a higher risk of leakage [1]. That is why, most of the already published surgical techniques focus on the preservation of the stomach. In our case, the distal location of the esophageal tumor as well as the difficult dissection of the stomach vessels due to the chronic inflammation in the region made the interposition of a gastric tube impossible. None of the anastomoses were compromised by a leakage and no major complications developed postoperatively. In our opinion, when meticulous surgical technique is used, there is no problem with the use of colon as a substitute in esophageal resections.

\section{Conclusion}

Combined multiorgan resections may be sometimes an acceptable alternative in selected patients. Meticulous surgical technique, multidisciplinary management and close observation at regular postoperative intervals are necessary prerequisites for satisfactory long-term results in such cases.

Open Access This article is distributed under the terms of the Creative Commons Attribution Noncommercial License which permits any noncommercial use, distribution, and reproduction in any medium, provided the original author(s) and source are credited.

\section{References}

1. Ikeda M, Hasegawa K, Akamatsu N, Minagawa M, Imamura H, Sugawara $\mathrm{Y}$ et al (2006) Pancreaticoduodenectomy after esophageal and gastric surgery preserving right gastroepiploic vessels. Arch Surg 141:205-208 doi:10.1001/archsurg.141.2.205

2. Nagano Y, Sekido H, Matsuoi K, Ohtsuki K, Gorai K, Kunisaki C et al (2005) Successful pancreatoduodenectomy for carcinoma of the ampulla of vater after esophagectomy with remnant gastrectomy. Hepatogastroenterology 52:933-935

3. Uehara H, Kondo S, Hirano S, Ambo Y, Tanaka E, Katoh H (2004) Gastric tube-preserving pancreatoduodenectomy after esophagectomy and reconstruction using the gastric tube: report of a case. Surg Today 34:623-625 doi:10.1007/s00595-004-2767-8

4. Kurosaki I, Hatakeyama K, Nihei K, Suzuki T, Tsukada K (2000) Thoracic esophagectomy combined with pylorus-preserving pancreatoduodenectomy in a one-stage procedure: report of a case. Surg Today 30:168-172 doi:10.1007/s005950050036

5. Mafune K, Tanaka Y, Ma YY, Takubo K (1995) Synchronous cancers of the esophagus and the ampulla of Vater after distal gastrectomy: successful removal of the esophagus, gastric remnant, duodenum, and pancreatic head. J Surg Oncol 60:277-281 doi:10.1002/jso.2930600412 